\title{
Preliminary results following the use of a fixed combination of timolol-brimonidine in patients with ocular hypertension and primary open-angle glaucoma
}

\author{
Dimitris Papaconstantinou' \\ Ilias Georgalas ${ }^{2}$ \\ Nikolaos Kourtis' \\ Christos Pitsas' \\ Efthimios Karmiris' \\ Chrysanthi Koutsandrea' \\ loannis Ladas' \\ Gerasimos Georgopoulos' \\ 'Department of Ophthalmology, \\ "G Gennimatas" Hospital of Athens, \\ University of Athens, Athens, Greece; \\ 2Department of Ophthalmology, \\ "G Gennimatas" Hospital of Athens, \\ NHS, Athens, Greece
}

\begin{abstract}
Purpose: The purpose of this prospective study was to evaluate the efficacy in intraocular pressure (IOP) control and the tolerance of a topically administered fixed combination of timololbrimonidine in 50 patients with ocular hypertension and primary open-angle glaucoma.

Methods: After determining a baseline IOP, the fixed combination timolol-brimonidine was used twice daily for two months, while IOP, ophthalmic signs, and/or symptoms were monitored.

Results: The mean IOP value was decreased from $23.09 \mathrm{~mm} \mathrm{Hg}( \pm 1.98 \mathrm{SD})$ to $17.46 \mathrm{~mm} \mathrm{Hg}$ ( $\pm 1.47 \mathrm{SD}$ ) during the 1 st month (paired Student's $\mathrm{t}$ test $=9.88 \kappa \alpha \mathrm{p}<0.001$ ), and to $17.51 \mathrm{~mm} \mathrm{Hg}( \pm 1.43 \mathrm{SD})$ in the 2 nd month. Between the 1 st and 2 nd month, no statistical difference was observed (paired Student's t test $=0.02 \kappa \alpha \mathrm{p}>0.1$ ). In $8 \%$ of the patients during the 1 st month and $10 \%$ of patients in the 2nd month, some ophthalmic signs were observed, while only mild ophthalmic symptoms were reported in $6 \%$ and $8 \%$ of the patients, respectively.

Conclusions: In conclusion, the fixed combination of timolol-brimonidine has a satisfactory IOP-lowering effect without any serious side effects due to the topical use.
\end{abstract}

Keywords: fixed combination $0.2 \%$ brimonidine $-0.5 \%$ timolol, ocular hypertension, primary open-angle glaucoma

\section{Introduction}

Intraocular pressure (IOP) is the most important prognostic risk factor, associated with the gradual vision loss in glaucoma. ${ }^{1,2}$ Lowering IOP has been proved to contribute effectively in delaying the development of glaucoma in patients with ocular hypertension, and also in delaying the progression of established glaucoma. ${ }^{3}$

The medications already in use decrease the IOP effectively, giving the opportunity to achieve the target IOP even with monotherapy. Nevertheless, many patients with glaucoma do not reach target IOPs with only one medication, which makes necessary the introduction of a second or even a third IOP-lowering drug. According to the Ocular Hypertension Treatment Study, after five years of IOP-lowering medical treatment, $40 \%$ of the patients need at least two drugs for effective IOP control.

Beta-adrenergic antagonists and alpha-adrenergic agonists are two commonly used categories of IOP-lowering medications. Timolol is a nonselective $\beta$-blocker, which lowers IOP by decreasing aqueous humor production, and brimonidine is an $\alpha$-agonist which has a double action by decreasing aqueous humor production and, also, by increasing aqueous humor outflow via the uveoscleral pathway. Various studies demonstrated a greater decrease in IOP with the fixed combination timolol-brimonidine compared with the use of each drug separately..$^{5-8}$ The purpose of this study is to
Correspondence: Dimitris Papaconstantinou Consultant Opthalmic Surgeon,

G. Gennimatas Hospital, Mesogeion Ave, Athens, Greece

Tel +30 2l 0776800

Email dpapaconstantinou@hotmail.com 
evaluate the efficacy of timolol-brimonidine in controlling IOP and its tolerance in patients with ocular hypertension and primary open-angle glaucoma (POAG).

\section{Methods}

\section{Patients}

This study evaluates the efficacy and tolerance of the fixed combination timolol-brimonidine in patients with ocular hypertension and POAG. For this purpose a two-month prospective study was conducted at the 1st University Eye Clinic of Athens.

Eligible patients had to have POAG or ocular hypertension and should have no prior antihypertensive medical treatment or only monotherapy. Key exclusion criteria included:

1. Active ocular infection or inflammation

2. Previous sensitivity to timolol or brimonidine

3. Unstable heart disease that could be negatively affected from the use of the $\beta$-blocker

4. History of dendritic ulcer, bullous keratopathy, or active corneal ulcer in the past three months

5. Corneal abnormalities that interfere with an accurate IOP measurement (eg, pterygium)

6. Retinal disease (eg, previous retinal detachment, diabetic retinopathy, or any other progressive disease)

7. Uses contact lens

8. Previous intraocular surgery or laser trabeculoplasty

9. Angle closure or secondary glaucoma

10. Disease processes such as blepharitis/chronic conjunctivitis.

The study was conducted in compliance with the Declaration of Helsinki (1996) and in accordance with institutional review board regulations. Each participating investigator received institutional review board approval and obtained written informed consent from patients before initiation of the study.

\section{Intervention}

The study was conducted at the following phases. At the beginning, took place a baseline examination in order to classify patients according to the aforementioned criteria, an IOP measurement and a detailed medical history.

Patients who didn't receive any medication had a baseline IOP measurement.

Patients already on medication at the baseline examination underwent a wash-out period followed by the baseline IOP measurement. Wash-out periods, according to the previous drug, were determined as:
- $\quad \beta$-blockers: two weeks

- $\alpha$-agonists: one week

- prostaglandin analogs: one week

- topical carbonic anhydrase inhibitors: one week

The baseline IOP measurement included a measurement in the morning, before $11 \mathrm{AM}$ on two different days of the same week. The mean IOP of these measurements was considered as the baseline IOP and was used for comparison of the results. At the same time a slit lamp and a fundus examination took place.

Afterwards, the fixed combination timolol-brimonidine was given and the dosage was determined as one drop every 12 hours at $8 \mathrm{AM}$ and $8 \mathrm{PM}$.

Follow-up study visits were scheduled one and two months after the initiation of therapy. During the follow-up examination, IOP measurement was performed approximately at the same time (11 AM), three hours after drug instillation.

Variables that were evaluated, were ophthalmic signs evaluated under a slit lamp (conjunctival secretions, examination of the conjunctiva and corneal epithelium), and tolerance of the drug (pain, burning, stinging).

\section{Results}

Patient ages varied from 39 to 83 years and the mean age was 63.5 years. Fifty patients were recruited: 26 were men and 24 women. Six patients had no prior antihypertensive treatment and 44 were already on medication which is shown in Table 1.

Forty-six patients completed the study. Four patients discontinued treatment for the following reasons: topical adverse events in one patient who developed epithelial damage of the cornea and blurring of vision, and uncontrolled IOP in three patients. Patients who discontinued treatment

Table I Intraocular pressure-lowering drugs used before initiation of the fixed combination timolol-brimonidine and their percentage

\begin{tabular}{lll}
\hline Medication & No of patients & $\mathbf{( \% )}$ \\
\hline No medication & 6 & $12 \%$ \\
Brimonidine & 3 & $6 \%$ \\
Timolol & 5 & $10 \%$ \\
Latanoprost & 7 & $14 \%$ \\
Travoprost & 2 & $4 \%$ \\
Bimatoprost & 2 & $4 \%$ \\
Dorzolamide & 2 & $4 \%$ \\
Timolol/Dorzolamide & 14 & $28 \%$ \\
Latanoprost/Timolol & 9 & $18 \%$ \\
Total & 50 & \\
\hline
\end{tabular}


were included in data analysis with the measurements taken until the time of withdrawal.

\section{Intraocular pressure}

In order to verify the wash-out effect in the group of patients with prior antihypertensive treatment, we compared the mean IOP at the baseline examination before the wash-out period $(19.61 \mathrm{mmHg} \pm 1.53 \mathrm{SD})$ with the mean IOP after this period (22.68 mmHg, $\pm 1.71 \mathrm{SD})$. The statistical analysis showed a significant difference (paired Student's t test $=5.86$ and $\mathrm{p}=0.001$ ).

The baseline mean IOP decreased from $23.09 \mathrm{mmHg}$ $( \pm 1.98 \mathrm{SD})$ to $17.46 \mathrm{mmHg}( \pm 1.47 \mathrm{SD})$ at the $1 \mathrm{st}$ month examination. The statistical analysis showed a significant difference (paired Student's t test $=9.88$ and $p<0.001$ ). At the 2nd month examination the mean IOP was $17.51 \mathrm{mmHg}$ $( \pm 1.43 \mathrm{SD})$ and the statistical analysis demonstrated no significant difference between the 1st and 2nd month (paired Student's t test $=0.02$ and $\mathrm{p}>0.1$ ). Figure 1 shows the variation of IOP.

\section{Tolerance}

The evaluation of the drug tolerance included patient symptoms and slit lamp examination at the baseline examination and at the end of the 1 st and 2 nd months.

With the slit lamp examination, particular attention was given to the cornea, conjunctiva, and presence of secretions. Among the 50 patients, four in the 1 st month (8\%) and five in the 2 nd month (10\%) presented with an ophthalmic sign, which is given in detail in Table 2 . We note that certain patients presented with more than one ophthalmic sign.

During the follow-up visits, the patients completed a questionnaire about the appearance of symptoms such as stinging, burning, pain and their classification as mild, moderate,

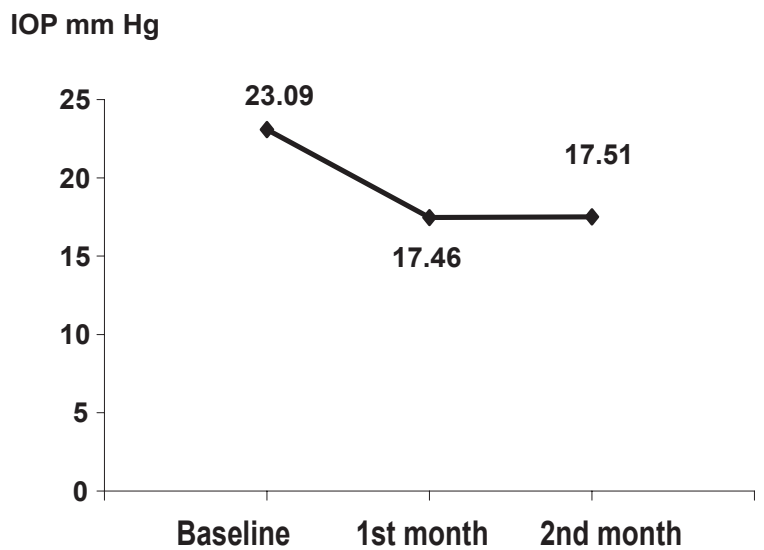

Figure I Mean intraocular pressure (IOP) change.
Table 2 Number of eyes that presented the specific sign at the Ist and/or 2nd month, with no prior involvement

\begin{tabular}{lll}
\hline Ophthalmic signs & Ist month & 2nd month \\
\hline Conjunctival secretions & 3 & 3 \\
Conjunctiva & 4 & 4 \\
Corneal epithelial disease & I & 0 \\
Total number of patients & $4^{*}$ & $5^{* *}$
\end{tabular}

Notes: *One patient had three signs and one patient had two signs; ${ }^{* *}$ Two patients had two signs.

and serious. None of the 50 patients mentioned any moderate or serious symptom during the follow-up visits. Three patients at the end of the 1 st month $(6 \%)$ and four patients at the end of the 2 nd month ( $8 \%$ ) mentioned mild symptoms (Table 3 ). It should be mentioned that no patient reported pain at the end of the 2 nd month.

\section{Discussion}

This study showed that there is a statistically significant difference of the IOP before and after the initiation of the fixed combination timolol-brimonidine in our group of patients, not related to their prior antihypertensive treatment. The mean baseline IOP decreased from $23.09 \mathrm{mmHg}( \pm 1.98 \mathrm{SD})$ to $17.46 \mathrm{mmHg}( \pm 1.47 \mathrm{SD})$ at the end of the 1 st month. There have been similar results of the IOP-lowering effect in other studies investigating the effect of the fixed combination timolol-brimonidine vs brimonidine alone, vs a $\beta$-blocker, or vs the fixed combination dorzolamide-timolol. ${ }^{5-10}$ Our study agrees with all these relevant studies on the fact that the fixed combination timolol-brimonidine twice daily offers a better control of IOP in cases of ocular hypertension and POAG when compared with a monotherapy. An important factor contributing to the more efficient IOP control with this fixed combination could be that the dosage, twice daily, results in better patient compliance. This would be more difficult with the use of two different eyedrops, each one of them given twice daily.

In addition, only four patients at the 1 st month (8\%) and five at the 2 nd (10\%) presented with an ophthalmic sign and none had a moderate or serious symptom during the followup visits. In contrast, the incidence of adverse events is higher with the use of timolol and brimonidine separately and often

Table 3 Eyes that presented with symptoms at Ist and 2nd month, but were not present at the baseline examination

\begin{tabular}{llll}
\hline Months & Stinging & Burning & Pain \\
\hline Ist month & 2 & 0 & I \\
2nd month & 2 & 2 & 0 \\
\hline
\end{tabular}


leads to discontinuation of treatment. ${ }^{5,6}$ Conclusively, we postulate a significant safety limit with the use of the fixed combination timolol-brimonidine and, also, a low incidence of adverse events. This is in accordance with the current literature and recent studies. , $, 6,11^{-1}$

\section{Conclusions}

The fixed combination timolol-brimonidine is administered twice daily and exhibits a significant IOP-lowering effect compared with any previous antihypertensive treatment. Also, its topical use isn't associated with any serious side effects which results in high safety and better patient compliance with this specific antihypertensive treatment.

\section{Disclosure}

The authors report no conflicts of interest in this work.

\section{References}

1. Nouri-Mahdavi K, Hoffman D, Coleman AL, et al; Advanced Glaucoma Intervention Study. Predictive factors for glaucomatous visual field progression in the Advanced Glaucoma Intervention Study. Ophthalmology. 2004;111:1627-1635.

2. Bengtsson B, Leske MC, Hyman L, Heijl A; Early Manifest Glaucoma Trial Group. Fluctuation of intraocular pressure and glaucoma progression in the early manifest glaucoma trial. Ophthalmology. 2007;114:205-209.
3. Heijl A, Leske MC, Bengtsson B, Hyman L, Bengtsson B, Hussein M; Early Manifest Glaucoma Trial Group. Reduction of intraocular pressure and glaucoma progression: results from the Early Manifest Glaucoma Trial. Arch Ophthalmol. 2002;120:1268-1279.

4. Gordon MO, Beiser JA, Brandt JD, et al. The Ocular Hypertension Treatment Study: baseline factors that predict the onset of primary open-angle glaucoma. Arch Ophthalmol. 2002;120:714-720.

5. Sherwood MB, Craven ER, Chou C, DuBiner HB, Batoosingh AL, Schiffman RM, Whitcup SM. Twice-daily $0.2 \%$ brimonidine- $0.5 \%$ timolol fixed-combination therapy vs monotherapy with timolol or brimonidine in patients with glaucoma or ocular hypertension: a 12-month randomized trial. Arch Ophthalmol. 2006;124:1230-1238.

6. Goñi FJ; Brimonidine/Timolol Fixed Combination Study Group. 12-week study comparing the fixed combination of brimonidine and timolol with concomitant use of the individual components in patients with glaucoma and ocular hypertension. Eur J Ophthalmol. 2005; 15:581-590.

7. Craven ER, Walters TR, Williams R, Chou C, Cheetham JK, Schiffman R; Combigan Study Group. Brimonidine and timolol fixed-combination therapy versus monotherapy: a 3-month randomized trial in patients with glaucoma or ocular hypertension. J Ocul Pharmacol Ther. 2005; 21:337-348

8. Konstas AG, Katsimpris IE, Kaltsos K, et al. Twenty-four-hour efficacy of the brimonidine/timolol fixed combination versus therapy with the unfixed components. Eye. 2008;22:1391-1397.

9. Arcieri ES, Arcieri RS, Pereira AC, Andreo EG, Finotti IG, Sá Filho WF. Comparing the fixed combination brimonidine-timolol versus fixed combination dorzolamide-timolol in patients with elevated intraocular pressure. Curr Med Res Opin. 2007;23:683-689.

10. Frampton JE. Topical brimonidine $0.2 \%$ /timolol $0.5 \%$ ophthalmic solution: in glaucoma and ocular hypertension. Drugs Aging. 2006;23:753-761.

11. Alvarado JA. Reduced ocular allergy with fixed-combination $0.2 \%$ brimonidine-0.5\% timolol. Arch Ophthalmol. 2007;125:717. 